\title{
A SUPERELEMENT-BASED METHOD FOR COMPUTING UNSTEADY THREE-DIMENSIONAL POTENTIAL FLOWS IN HYDRAULIC TURBOMACHINES
}

\author{
N. P. KRUYT*, B. P. M. VAN ESCH AND J. B. JONKER \\ Department of Mechanical Engineering, University of Twente, The Netherlands
}

\begin{abstract}
SUMMARY
A numerical method is presented for the computation of unsteady, three-dimensional potential flows in hydraulic pumps and turbines. The superelement method has been extended in order to eliminate slave degrees of freedom not only from the governing Laplace equation, but also from the Kutta conditions. The resulting superelement formulation is invariant under rotation. Therefore the geometrical symmetry of the flow channels in the rotor can be exploited. This makes the method especially suitable to performing fully coupled computations of the unsteady flow phenomena in both rotor and stator, the so-called rotor-stator interaction.

The developed numerical method is used to simulate the unsteady flow in an industrial mixed-flow pump. Two types of simulation are considered: one in which unsteady wakes behind the trailing edges of the rotor blades are taken into account and one in which these are neglected. Results are given that show the importance of unsteady flow phenomena. However, the computed head-capacity curve is hardly influenced by whether or not unsteady wakes are taken into account. Copyright (C) 1999 John Wiley \& Sons, Ltd.
\end{abstract}

KEY WORDS finite-element method; superelements; potential flow; turbomachines; pumps; rotor-stator interaction

\section{INTRODUCTION}

The design of hydraulic turbomachines, such as pumps and water turbines, has reached the stage where improvements of their performance can only be achieved through a detailed understanding of the internal flow phenomena. The prediction of the flow in such equipment is very complicated due to the three-dimensionality of the flow and the highly curved passages in the rotor. Furthermore, turbomachines show unsteady flow behaviour, especially at off-design conditions, as a result of the interaction between rotor and stator. Considering these complexities, computer simulations of the flow are becoming increasingly important.

Over the past few years there has been a tendency towards the development of numerical methods based on the Navier-Stokes equations in order to account for viscous effects like wakes, boundary layers, recirculation zones and separation bubbles. Examples in the field of turbomachines are given in References 1 and 2. An open problem in such computations is the choice of an appropriate turbulence model and boundary conditions, since the standard turbulence models appear not to be adequate in rotating systems. ${ }^{3}$ Furthermore, the computer time needed to

* Correspondence to: Dr. N. P. Kruyt, Department of Mechanical Engineering, University of Twente, PO Box 217, 7500 AE Enschede, The Netherlands.

CCC 1069-8299/99/060381-17\$17.50

Copyright (C) 1999 John Wiley \& Sons, Ltd.

Received 25 November 1996

Accepted 15 September 1998 
compute the flow through a single rotor channel is very large. Therefore these methods are of limited suitability as part of a design tool.

Fortunately, the physical model can be simplified considerably without losing its overall validity. Usually the flow conditions in hydraulic turbomachines are such that (i) the fluid can be considered as incompressible (small Mach number), (ii) the bulk of the fluid can be regarded as inviscid, since viscous forces are negligible when compared to inertia forces (large Reynolds number), and (iii) the flow enters the rotor free of vorticity. For an inviscid bulk flow this means that the flow remains irrotational. Combining these assumptions leads to the incompressible potential flow model.

Many investigators have presented numerical methods for computing potential flows inside a rotor channel in two or three dimensions $s^{4-6}$ and in complete rotor-stator configurations in two dimensions. ${ }^{7-10}$ In order to impose the Kutta condition of smooth flow at the blades' trailing edges, they either superimpose a number of subpotentials (reflecting unit flowrate, unit rotation and line vortices of unit strength, shed from points at the trailing edges) or determine the circulations iteratively.

In this paper an efficient method is proposed for computing the unsteady, three-dimensional potential flow inside complete rotor-stator configurations, which takes into account the varying circulation along the blades' span. The computation of such flows is of great importance to the study of rotor-stator interaction. The developed method is based on the well-known superelement method. It is extended here in order to take into account the Kutta conditions at the blades' trailing edges. Two types of computations are distinguished: quasi-steady computations in which unsteady wakes are neglected and unsteady computations in which these are taken into account.

\section{FLOW MODEL}

\section{Basic equations}

The three-dimensional flow of an inviscid, incompressible fluid is considered. Assuming the flow to be irrotational in the absolute frame of reference, the problem can be formulated in terms of a velocity potential $\phi$. The velocity vector $\underline{v}$ can then be written as

$$
\underline{v}=\nabla \phi
$$

With (1) the continuity equation reduces to the Laplace equation

$$
\nabla^{2} \phi=0
$$

and the Navier-Stokes equations reduce to the unsteady Bernoulli equation

$$
\frac{\partial \phi}{\partial t}+\frac{1}{2} \underline{v} \cdot \underline{v}+\frac{p}{\rho}+g z=c(t)
$$

Here $t$ denotes time, $p$ static pressure, $\rho$ density and $c(t)$ a constant which only depends on $t$. Note that the change in total pressure is due to the time derivative of the potential. Unsteadiness is introduced by the rotating motion of the rotor blades and by the interaction with the stator. 


\section{Kutta conditions}

In the potential flow model the blade circulations are indeterminate. ${ }^{11}$ The Kutta condition of smooth flow from the trailing edges (te) of the rotor blades is used to determine these circulations. The Kutta conditions describe a viscous effect within an inviscid theory. They require that the flow leaves 'smoothly' from the trailing edges. Here the Kutta conditions are formulated as

$$
\left.\frac{\partial \phi}{\partial n}\right|_{\mathrm{te}}=\left.(\underline{\Omega} \times \underline{r}) \cdot \underline{n}\right|_{\mathrm{te}}
$$

where $\underline{\Omega}$ is the angular velocity of the rotor, $\underline{r}$ is the position vector and $\underline{n}$ is the outward unit normal vector.

\section{Wakes}

For three-dimensional blades the circulation will in general vary along the blade span. In unsteady flow the circulation will also vary in time. From Kelvin's circulation theorem for inviscid flow ${ }^{11}$ it follows that a time-dependent circulation around a blade must result in a continuous shedding of vortices with strength equal to the change in bound vorticity. The vortex sheet which is formed is called the wake (see Figure 1).

In potential flow theory, wakes are modelled by means of jumps (discontinuities) in the potential on wake surfaces

$$
\phi^{+}\left(s_{1}, s_{2}\right)=\phi^{-}\left(s_{1}, s_{2}\right)+\gamma\left(s_{1}, s_{2}\right)
$$

where ' + ' and ' - ' denote the upper and lower sides of the wake, $s_{1}$ and $s_{2}$ are co-ordinates along the wake ( $s_{1}$ is in the streamwise direction) and $\gamma\left(s_{1}, s_{2}\right)$ is the potential jump distribution. The blade circulation $\Gamma\left(s_{2}\right)$ at spanwise station $s_{2}$ is related to the potential jump distribution by

$$
\Gamma\left(s_{2}\right)=\gamma\left(0, s_{2}\right)
$$

The nature of the potential jump distribution depends on whether the flow is considered as quasisteady or unsteady.

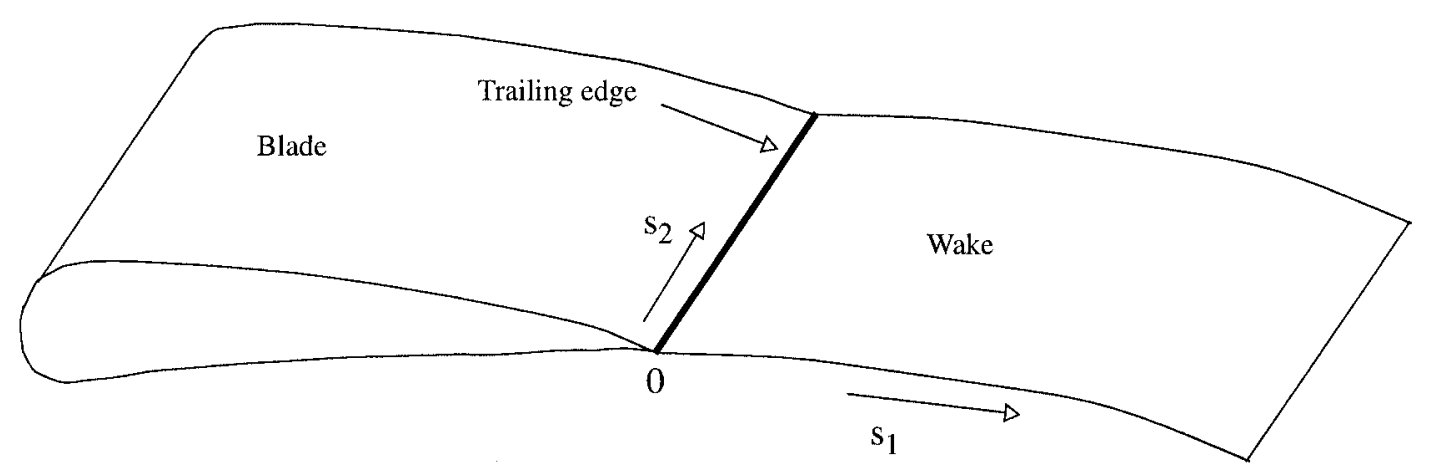

Figure 1. Representation of a wake behind a blade. Co-ordinate $s_{1}$ is in streamwise direction 
In unsteady simulations the evolution of the potential jump distribution follows from the unsteady Bernoulli equation (3)

$$
\frac{\partial \gamma}{\partial t}=\frac{\partial}{\partial t}\left(\phi^{+}-\phi^{-}\right)=-\frac{1}{2}\left(\left|\nabla \phi^{+}\right|^{2}-\left|\nabla \phi^{-}\right|^{2}\right)
$$

since the pressure is continuous across the wake. After linearization this results in

$$
\frac{\partial \gamma}{\partial t}+V_{s_{1}} \frac{\partial}{\partial s_{1}}\left(\phi^{+}-\phi^{-}\right)=0
$$

This means that the potential jump in the wake is convected downstream with the mean velocity $V_{s_{1}}$ along the wake. The distribution of the potential jump is time-dependent and can only be determined by performing a number of computations at successive time steps.

In quasi-steady simulations, in which the convection of vortices in the wake is neglected, the potential jump over the wake surface is constant in the streamwise direction

$$
\gamma\left(s_{1}, s_{2}\right)=\Gamma\left(s_{2}\right)
$$

Summarizing, in quasi-steady computations (without unsteady wakes) the potential jump distribution in the wake is given by (9), while in unsteady computations (with unsteady wakes) the potential jump distribution satisfies (8).

\section{Boundary conditions}

On the inlet and outlet surfaces of the turbomachine, a uniform normal velocity is prescribed

$$
\frac{\partial \phi}{\partial n}= \pm \frac{Q}{A}
$$

where $Q$ is the flowrate and $A$ is the area of the surface.

At the blade surfaces (both pressure and suction sides) the Neumann boundary condition takes the form

$$
\frac{\partial \phi}{\partial n}=(\underline{\Omega} \times \underline{r}) \cdot \underline{n}
$$

At the hub and the shroud of the rotor and at the stator walls, the normal velocity vanishes:

$$
\frac{\partial \phi}{\partial n}=0
$$

Wakes are present behind trailing edges. These wakes are a result of both non-uniform blade loading (variations of the circulation along the blade's span) and time-dependent variations of 
the blade circulations. Within the potential flow model, wakes are modelled by the boundary conditions

$$
\begin{aligned}
\phi^{+}\left(s_{1}, s_{2}\right) & =\phi^{-}\left(s_{1}, s_{2}\right)+\gamma\left(s_{1}, s_{2}\right) \\
\frac{\partial}{\partial n} \phi^{+}\left(s_{1}, s_{2}\right) & =-\frac{\partial}{\partial n} \phi^{-}\left(s_{1}, s_{2}\right)
\end{aligned}
$$

The second equation of (13) states that the normal velocity is continuous on the wake surface. Note that wakes should coincide with stream surfaces. In general an iterative method is needed to meet this requirement.

\section{ROTOR-STATOR INTERFACE}

When considering configurations of complete pumps or turbines, special care has to be taken of the presence of both rotating and stationary parts. In order to achieve this without having to create a new mesh for each time step (as was done in Reference 8), the rotor and the stator are separated by a cylindrical or conical surface, the so-called rotor-stator interface. Connections between nodes at both sides of this interface are changed over time. In this way the rotor is allowed to rotate freely while 'sliding' along the stator.

Both the rotor and stator part have a separate co-ordinate system attached to them. Time deratives of the potential, which are necessary to compute the pressure from the unsteady Bernoulli equation, are determined relative to these co-ordinate systems by a central difference scheme in time. Since the material derivative and the gradient operator are invariant under a transformation to a rotating co-ordinate system, it follows that

$$
\left.\frac{\partial \phi}{\partial t}\right|_{R}+\left.\underline{w} \cdot \nabla \phi \equiv \frac{D \phi}{D t}\right|_{R}=\frac{D \phi}{D t} \equiv \frac{\partial \phi}{\partial t}+\underline{v} \cdot \nabla \phi
$$

The subscript $R$ indicates that the time derivative of the potential is taken relative to the rotating frame of reference. The velocity in the rotating frame of reference $\underline{w}$ is defined by

$$
\underline{v}=\underline{w}+\underline{\Omega} \times \underline{r}
$$

Using these equations, the unsteady Bernoulli equation in the rotating frame of reference becomes

$$
\left.\frac{\partial \phi}{\partial t}\right|_{R}-(\underline{\Omega} \times \underline{r}) \cdot \underline{v}+\frac{1}{2} \underline{v} \cdot \underline{v}+\frac{p}{\rho}+g z=c(t)
$$

Similarly, equation (8) that describes the evolution of the potential jump on the wake can be rewritten as

$$
\left.\frac{\partial \gamma}{\partial t}\right|_{R}+W_{s_{1}} \frac{\partial}{\partial s_{1}}\left(\phi^{+}-\phi^{-}\right)=0
$$

where $W_{s_{1}}$ is the mean velocity along the wake in the rotating frame of reference.

This method is identical to that employed in References 9 and 10. 


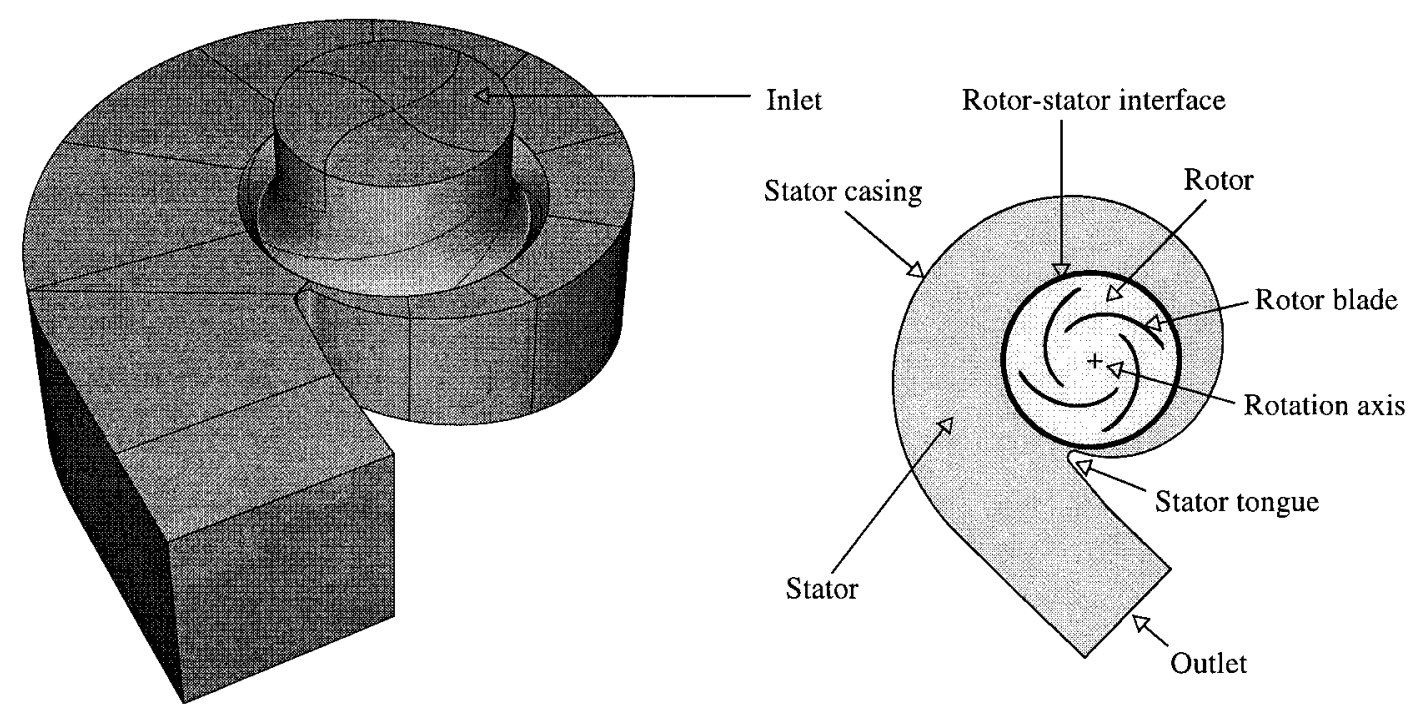

Figure 2. Example of a pump geometry divided into blocks and cross-section of pump

\section{MULTI-BLOCK APPROACH}

The presence of a rotor and a stator part with their separate co-ordinate systems naturally suggests using a multi-block approach. In such a multi-block approach the flow region of interest is divided into subdomains or blocks, all having a topologically cubic shape. The subdomains are non-overlapping, with nodal coincidence at the interfaces. For a free rotor computation one block will usually suffice, although a division into more blocks is possible. However, for a flow computation inside a complete pump or turbine (rotor and stator) a number of blocks is required (see Figure 2).

An advantage of the multiblock approach is the greater ease in creating a good mesh for the complex three-dimensional geometries that are considered here. It also constitutes an important component of the numerical method that is described in the next Section.

In the multiblock approach additional boundary conditions have to be formulated that apply to the artificial internal boundaries between blocks. The velocity field at these internal boundaries should be continuous. Therefore the values of the potential at corresponding nodes can differ only by a fixed amount and the normal velocities are opposite. This means that the boundary conditions for such internal boundaries are the same as those for wakes (see equation (13)), with $\gamma$ constant. Periodic boundary conditions, for a free rotor computation, are also of this type.

\section{NUMERICAL METHOD}

\section{Outline}

The flow field is solved by means of a finite element method using an extension of the superelement technique. ${ }^{12}$ In the superelement technique internal degrees of freedom (DOFs) are eliminated from the discretized governing (Laplace) equation. The extension of the superelement 
method developed here deals with an analogous elimination of the internal DOFs from the discretized Kutta conditions. The method consists of two steps:

- elimination of internal DOFs from the system of equations (Laplace equation and Kutta conditions), for all blocks separately; this leads to the formulation of the superelements.

- assemblage of the superelements. After solving the resulting global system of equations, the previously eliminated DOFs are obtained.

\section{Superelement formulation: elimination step}

For each block, the Laplace equation for the velocity potential together with the natural and essential boundary conditions (if any) is discretized according to the standard finite-element method, resulting in a system of linear equations

$$
[L]\{\phi\}=\{F\}+\{R\}
$$

where $[L]$ is a positive-definite matrix reflecting the discretized Laplace operator, $\{\phi\}$ is the vector of DOFs and $\{F\}$ is the vector corresponding to flowrates through block boundaries resulting from Neumann-type boundary conditions. Vector $\{R\}$ is related to (unknown) flowrates at internal block boundaries.

For each block, the discretized Kutta conditions (equation (4)) can be expressed in terms of potential values in the block using the modified gradient operator $[K]$ ( $K$ stands for Kutta):

$$
[K]\{\phi\}=\left\{\left.(\underline{\Omega} \times \underline{r}) \cdot \underline{n}\right|_{\mathrm{te}}\right\}
$$

Using the values of the potential, operator $[K]$ gives the normal velocities at the trailing edges. The basic idea of the superelement technique is to express (18) and (19) in terms of DOFs at internal block boundaries (called the 'master' DOFs), by eliminating the remaining interior 'slave' DOFs. For this purpose (18) and (19) are partitioned as follows:

$$
\begin{gathered}
{\left[\begin{array}{cc}
{\left[L^{\mathrm{ss}}\right]} & {\left[L^{\mathrm{sm}}\right]} \\
{\left[L^{\mathrm{ms}}\right]} & {\left[L^{\mathrm{mm}}\right]}
\end{array}\right]\left\{\begin{array}{c}
\left\{\phi^{\mathrm{s}}\right\} \\
\left\{\phi^{\mathrm{m}}\right\}
\end{array}\right\}=\left\{\begin{array}{c}
\left\{F^{\mathrm{s}}\right\} \\
\left\{F^{\mathrm{m}}\right\}+\left\{R^{*}\right\}
\end{array}\right\}} \\
{\left[\left[K^{\mathrm{s}}\right] \quad\left[K^{\mathrm{m}}\right]\right]\left\{\begin{array}{c}
\left\{\phi^{\mathrm{s}}\right\} \\
\left\{\phi^{\mathrm{m}}\right\}
\end{array}\right\}=\left\{\left.(\underline{\Omega} x \underline{r}) \cdot \underline{n}\right|_{\mathrm{te}}\right\}}
\end{gathered}
$$

Superscripts s and m denote 'slaves' and 'masters', respectively. Vector $\left\{R^{*}\right\}$ denotes the non-zero part of vector $\{R\}$ in (18).

By extracting the slave DOFs from (20) it follows that

$$
\left\{\phi^{\mathrm{s}}\right\}=\left[L^{\mathrm{ss}}\right]^{-1}\left\{F^{\mathrm{s}}\right\}-\left[L^{\mathrm{ss}}\right]^{-1}\left[L^{\mathrm{sm}}\right]\left\{\phi^{\mathrm{m}}\right\}
$$

The resulting 'super system' is obtained after substituting (22) into (20) and (21):

$$
\begin{aligned}
{\left[L^{\text {sup }}\right]\left\{\phi^{\mathrm{m}}\right\} } & =\left\{F^{\text {sup }}\right\}+\left\{R^{*}\right\} \\
{\left[K^{\text {sup }}\right]\left\{\phi^{\mathrm{m}}\right\} } & =\left\{G^{\text {sup }}\right\}
\end{aligned}
$$


where

$$
\begin{aligned}
{\left[L^{\mathrm{sup}}\right] } & =\left[L^{\mathrm{mm}}\right]-\left[L^{\mathrm{ms}}\right]\left[L^{\mathrm{ss}}\right]^{-1}\left[L^{\mathrm{sm}}\right] \\
{\left[K^{\mathrm{sup}}\right] } & =\left[K^{\mathrm{m}}\right]-\left[K^{\mathrm{s}}\right]\left[L^{\mathrm{ss}}\right]^{-1}\left[L^{\mathrm{sm}}\right] \\
\left\{F^{\mathrm{sup}}\right\} & =\left\{F^{\mathrm{m}}\right\}-\left[L^{\mathrm{ms}}\right]\left[L^{\mathrm{ss}}\right]^{-1}\left\{F^{\mathrm{s}}\right\} \\
\left\{G^{\mathrm{sup}}\right\} & =\left\{\left.(\underline{\Omega} x \underline{r}) \cdot \underline{n}\right|_{\mathrm{te}}\right\}-\left[K^{\mathrm{s}}\right]\left[L^{\mathrm{ss}}\right]^{-1}\left\{F^{\mathrm{s}}\right\}
\end{aligned}
$$

Note that column $i$ of $-\left[L^{\mathrm{ms}}\right]\left[L^{\mathrm{ss}}\right]^{-1}$ in (24) can be interpreted as the values of the slave potentials corresponding to master DOF $i$ equal to 1 and all other master DOFs equal to zero. Similarly, the term $\left[L^{\mathrm{ss}}\right]^{-1}\left\{F^{\mathrm{s}}\right\}$ in (25) represents the effect of Neumann boundary conditions on slave DOFs, while keeping the master DOFs equal to zero. Note that these potentials can be computed by simple backsubstitutions once the matrix $\left[L^{\text {ss }}\right]$ has been decomposed.

This Section describes how the reduced set of equations is obtained, in terms of DOFs at block interfaces only. In principle this procedure must be carried out for all blocks that form the entire geometry. However, an important observation is that the supermatrices and vectors are invariant under rotation for the scalar equations considered. Therefore the symmetry of the rotor can be exploited, as in general all rotor channels are geometrically identical. This means that the superelement formulation step has to be performed for the block(s) of a single rotor channel only! Furthermore, in a time-dependent computation, the superelement formulation step has to be carried out only once.

The assemblage of blocks, which can be regarded as superelements, is part of the second step. This is described in the following subsection.

\section{Assemblage of superelements: global solution step}

In the global solution step, the values of master DOFs of all participating blocks are determined by assembling and solving the global system of equations.

A complicating factor in the computation of the master DOFs is the fact that blade circulations and, as a consequence, the potential jumps at nodes on the wakes are still unknown. Therefore the values of blade circulations are regarded as additional variables to be determined along with the nodal DOFs (see also Reference 13). The vector of unknowns in the global problem is now denoted by

$$
\{u\}^{\mathrm{T}}=\left\{\phi_{1}, \ldots, \phi_{n_{\Phi}}, \Gamma_{1}, \ldots, \Gamma_{n_{\Gamma}}\right\}=\left\{\{\Phi\}^{\mathrm{T}}\{\Gamma\}^{\mathrm{T}}\right\}
$$

where $n_{\Phi}$ is the number of nodes in all block connections (coinciding nodes are counted as one), $n_{\Gamma}$ is the number of unknown blade circulations (i.e. the total number of nodes at trailing edges), $\{\Phi\}$ is the vector of unknown master DOFs for the potential and $\{\Gamma\}$ is the vector of unknown blade circulations.

The master DOFs can now be expressed in terms of global DOFs. Note that master DOFs may also involve potential jumps; see (13). These jumps are composed of known and unknown potential jumps (see also the Appendix).

All master DOFs of block $b$ are now written formally as

$$
\left\{\phi_{b}^{\mathrm{m}}\right\}=\left[T_{b}^{\Phi}\right]\{\Phi\}+\left[W_{b}\right]\{\Gamma\}+\left\{\gamma_{b}\right\}
$$


where

$\left[T_{b}^{\Phi}\right]$ is a matrix which gives the transformation of global equation numbers of nodal DOFs to the local numbering of master DOFs in block $b$. Each row contains exactly one non-zero coefficient, having the value 1

$\left[W_{b}\right]$ is a matrix which gives the equation numbers of global blade circulation DOFs for the masters of block $b$. It also accounts for the 'averaging' of potential jumps on the wakes (see the Appendix)

$\left\{\gamma_{b}\right\}$ are the known values of potential jumps at boundaries of block $b$. These jumps are present in computations including unsteady wakes (see the Appendix).

Similarly to the way in which element matrices and right-hand-side vectors are assembled to form the large system of equations, the superelement matrices and right-hand-side vectors of (23) are assembled into a global system of equations for the Laplace equation and for the Kutta conditions

$$
\begin{aligned}
& \sum_{b=1}^{n_{b}}\left[T_{b}^{\Phi}\right]^{\mathrm{T}}\left[L_{b}^{\mathrm{sup}}\right]\left\{\phi_{b}^{\mathrm{m}}\right\}=\sum_{b=1}^{n_{b}}\left[T_{b}^{\Phi}\right]^{\mathrm{T}}\left\{F_{b}^{\text {sup }}\right\} \\
& \sum_{b=1}^{n_{b}}\left[T_{b}^{\Gamma}\right]^{\mathrm{T}}\left[K_{b}^{\text {sup }}\right]\left\{\phi_{b}^{\mathrm{m}}\right\}=\sum_{b=1}^{n_{b}}\left[T_{b}^{\Gamma}\right]^{\mathrm{T}}\left\{G_{b}^{\mathrm{sup}}\right\}
\end{aligned}
$$

where matrices $\left[T_{b}^{\Gamma}\right]$ account for the transformation of the global blade circulation DOFs to the equation numbers of the local numbering of trailing edge nodes and $n_{b}$ is the total number of participating blocks. Note that the contribution of the vectors $\left\{R_{b}^{*}\right\}$ in (23) cancel out at internal block boundaries.

Substituting $\left\{\phi_{b}^{\mathrm{m}}\right\}$ from (27) into (28) gives the global system of equations

$$
\left[\begin{array}{ll}
{\left[M^{\Phi \Phi}\right]} & {\left[M^{\Phi \Gamma}\right]} \\
{\left[M^{\Gamma \Phi}\right]} & {\left[M^{\Gamma \Gamma}\right]}
\end{array}\right]\left\{\begin{array}{l}
\{\Phi\} \\
\{\Gamma\}
\end{array}\right\}=\left\{\begin{array}{l}
\left\{H^{\Phi}\right\} \\
\left\{H^{\Gamma}\right\}
\end{array}\right\}
$$

where

$$
\begin{aligned}
{\left[M^{\Phi \Phi}\right] } & =\sum_{b=1}^{n_{b}}\left[T_{b}^{\Phi}\right]^{\mathrm{T}}\left[L_{b}^{\text {sup }}\right]\left[T_{b}^{\Phi}\right] \quad\left[M^{\Phi \Gamma}\right]=\sum_{b=1}^{n_{b}}\left[T_{b}^{\Phi}\right]^{\mathrm{T}}\left[L_{b}^{\text {sup }}\right]\left[W_{b}\right] \\
{\left[M^{\Gamma \Phi}\right] } & =\sum_{b=1}^{n_{b}}\left[T_{b}^{\Gamma}\right]^{\mathrm{T}}\left[K_{b}^{\text {sup }}\right]\left[T_{b}^{\Phi}\right] \quad\left[M^{\Gamma \Gamma}\right]=\sum_{b=1}^{n_{b}}\left[T_{b}^{\Gamma}\right]^{\mathrm{T}}\left[K_{b}^{\text {sup }}\right]\left[W_{b}\right] \\
\left\{H^{\Phi}\right\} & =\sum_{b=1}^{n_{b}}\left[T_{b}^{\Phi}\right]^{\mathrm{T}}\left(\left\{F_{b}^{\text {sup }}\right\}-\left[L_{b}^{\text {sup }}\right]\{\gamma\}\right) \\
\left\{H^{\Gamma}\right\} & =\sum_{b=1}^{n_{b}}\left[T_{b}^{\Gamma}\right]^{\mathrm{T}}\left(\left\{G_{b}^{\text {sup }}\right\}-\left[K_{b}^{\text {sup }}\right]\{\gamma\}\right)
\end{aligned}
$$

Once the global system is solved, the solution for the potential for a block is obtained by first computing the values for the master DOFs (using (27)) and subsequently performing a back- 
substitution to determine the slave DOFs from (22). This procedure is carried out using the decomposed matrix $\left[L^{\mathrm{ss}}\right]$ which is stored on disc during the elimination step.

\section{Advantages of the new method}

Here the main advantages of the new method are summarized:

- The new method exploits the geometrical symmetry of the flow channels in the rotor. Only the superelements of a single flow channel need to be computed, since they are identical.

- The superelement matrices have to be computed only once during an unsteady computation.

- The Kutta conditions are imposed implicitly. Therefore the need no longer exists to determine a large number of subpotentials in order to impose the Kutta condition at all trailing edge nodes. This is especially important in three-dimensional computations, where the number of subpotentials increases rapidly, since the circulation varies along the span of the trailing edges.

- The elimination of internal DOFs results in a major reduction of computing time for unsteady computations, since these computations are performed with a greatly reduced number of DOFs.

\section{IMPLEMENTATION}

The developed numerical method has been implemented in a parametric hydraulic analysis system. Second-order accuracy for potential, velocities and pressures is obtained by employing linear elements in combination with the SPR-technique ${ }^{14}$ for the determination of the gradient of the potential, i.e. the velocity. In the global solution step the system of equations is solved using a direct method. The profile width of the sparse global matrix is reduced using a spectral renumbering technique. ${ }^{15,16}$

The various forms of modelling the wakes have been implemented (see also the Appendix).

\section{RESULTS}

An industrial mixed-flow pump of BW/IP Pumps Hengelo (Hengelo, The Netherlands) has been analysed using the developed hydraulic analysis system. In this Section results are presented concerning rotor-stator interaction and the overall head-capacity curve.

The dimensionless specific speed of the pump $n_{\Omega}=\left(\Omega Q_{\text {nom }}^{1 / 2}\right) /\left(g H_{\text {nom }}\right)^{3 / 4}$ equals $1 \cdot 6$, where $g$ is the acceleration due to gravity and $H_{\text {nom }}$ is the generated head at nominal flowrate $Q_{\text {nom }}$. The rotor of this pump consists of four blades $(Z=4)$. The radius and blade angle vary along the span of the (twisted) trailing edges. The geometry of the rotor and stator ${ }^{17}$ is shown in Figure 3. The stator of the pump is designed using the method of Pfleiderer, ${ }^{18}$ which is based on constant angular momentum of the fluid. The cross-sectional shape of the stator is trapezoidal. Note that in Figure 3 part of the stator wall is not shown: this gives a better view of the trapezoidal crosssectional shape of the stator.

In order to investigate rotor-stator interaction, time-dependent simulations have been carried out for this configuration for five flowrates until a periodic solution is obtained. The cases with and without unsteady wakes have both been considered. 
(a)

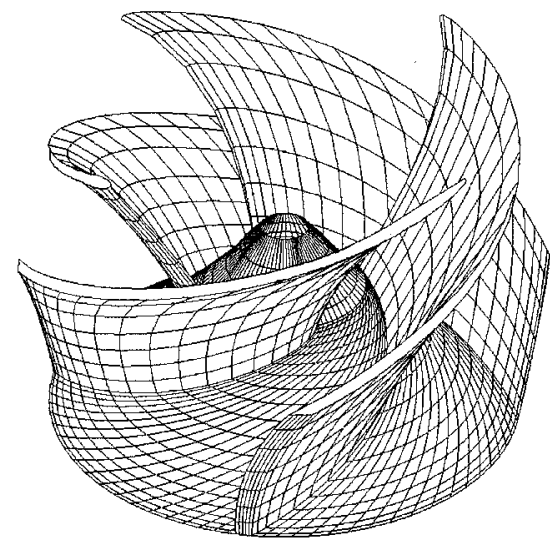

(c)

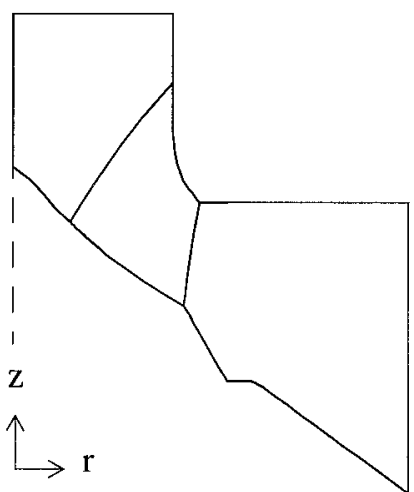

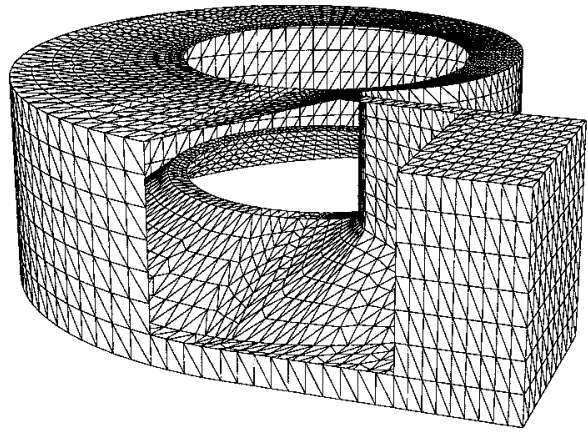

(b)

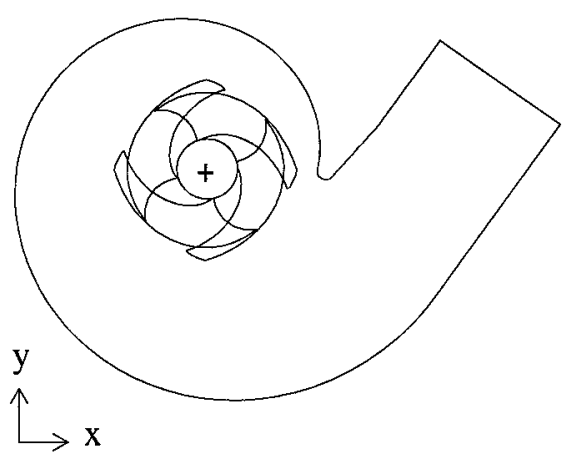

(d)

Figure 3. Geometry of BW/IP mixed-flow rotor and stator: (a) three-dimensional view of the impeller (shroud is not shown); (b) three-dimensional view of the stator (part of the stator wall is not shown); (c) cross-section of the pump; (d) projection of the pump on a plane perpendicular to the axis of rotation

Measures of the significance of the rotor-stator interaction are the effect of blade orientation $\theta$ with respect to the tongue of the stator on quantities like the spanwise average of the circulation around a blade and the flowrate through a rotor channel. The circulation around a blade is related to the energy transfer to the fluid. ${ }^{18}$

The orientation of a blade $\theta$ with respect to the tongue of the stator is not uniquely defined, since the trailing edges of the rotor blades are twisted and have a finite thickness. The tongue of the stator also has a finite thickness. Therefore the tongue position is indicated by a region. The orientation of a blade is counted as positive in the counter-clockwise direction.

The variation of spanwise-averaged circulation around a blade $\Gamma(\theta)$ with orientation $\theta$ is depicted in Figure 4 for various flowrates and for computations with and without unsteady wakes. The variations are made dimensionless with the average over all orientations. Note that this average is dependent on the flowrate. When unsteady wakes are not taken into account the variations are much larger (up to $60 \%$ ) than in computations that do take the unsteady wakes into account (up to $15 \%$ ). 


\section{Without unsteady wakes}

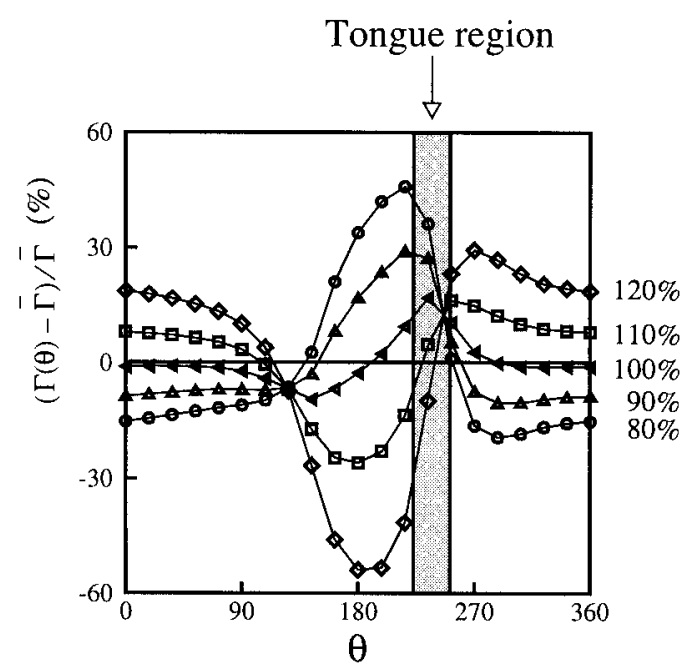

With unsteady wakes

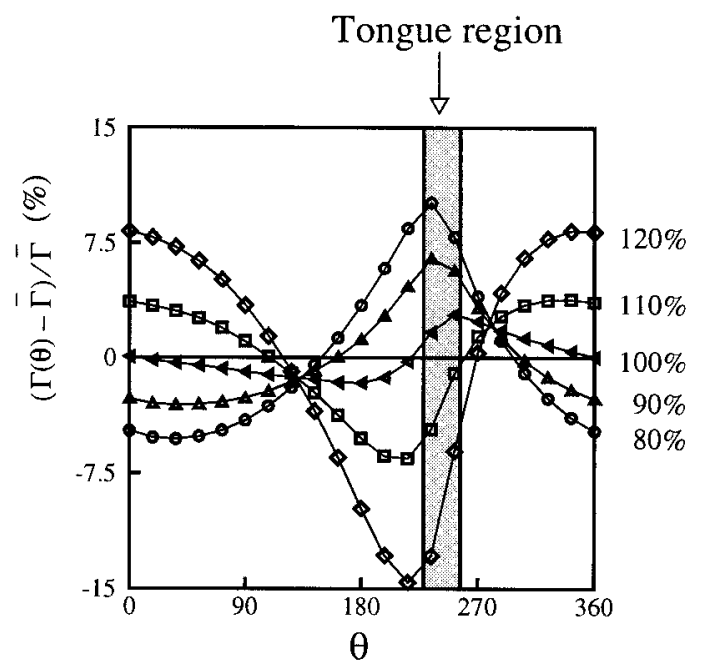

Figure 4. Comparison of the computations without and with unsteady wakes: variation of blade circulation $\Gamma(\theta)$ relative to the time-averaged circulation $\bar{\Gamma}$ as a function of orientation $\theta$ of the rotor blade, for flowrates of $80,90,100$, 110 and $120 \%$ of the nominal flowrate. The region of the stator tongue is marked

As can be observed in Figure 4, the unsteady effects are smallest for the nominal $(100 \%)$ flowrate. In both cases, with and without unsteady wakes, the variations increase with the deviation from the nominal flowrate. The variation of the circulation around the rotor, i.e. the sum of all blade circulations, is much smaller. For the simulations without unsteady wakes the order of magnitude of the variation is $3 \%$, while for the case with unsteady wakes it is $0.5 \%$. A varying circulation along the span of the rotor blades was observed in all cases. The order of magnitude of this spanwise variation is $15 \%$ (see also Reference 19).

Similarly, the variation of the flowrate in a rotor channel $q(\theta)$ with orientation $\theta$ is shown in Figure 5 for various total flowrates and for computations with and without unsteady wakes. Note that when the flowrates are divided evenly over all channels, the channel flowrate $q(\theta)$ equals $Q / Z$. Here it is also observed that computations without unsteady wakes predict larger variations (up to $40 \%$ ) than those that do take the unsteady wakes into account (up to $15 \%$ ). The unsteady effects are smallest for the nominal $(100 \%)$ flowrate. In both cases, with and without unsteady wakes, the variations increase with the deviation from the nominal flowrate.

The computations without unsteady wakes predict large variations of important flow quantities. Therefore the computations with unsteady wakes seem more realistic. However, no experimental evidence is currently available to support this assumption.

Finally, computed and measured dimensionless capacity-head curves are compared in Figure 6. Two computed head curves are shown, corresponding to the computations with and without unsteady wakes. The computed head curves are obtained by averaging the head over time, or orientation. As a reference the overall efficiency of the complete pump is also given. The difference in head between computations with and without unsteady wakes is small. The shape of the head-capacity curve is well predicted by the computations. The computations overestimate the head, since viscous losses are not accounted for in the basic potential flow model. 


\section{Without unsteady wakes}

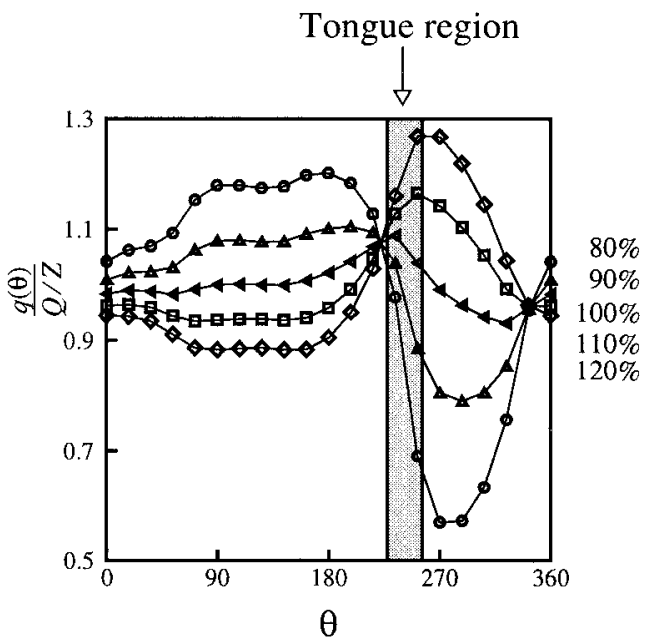

With unsteady wakes

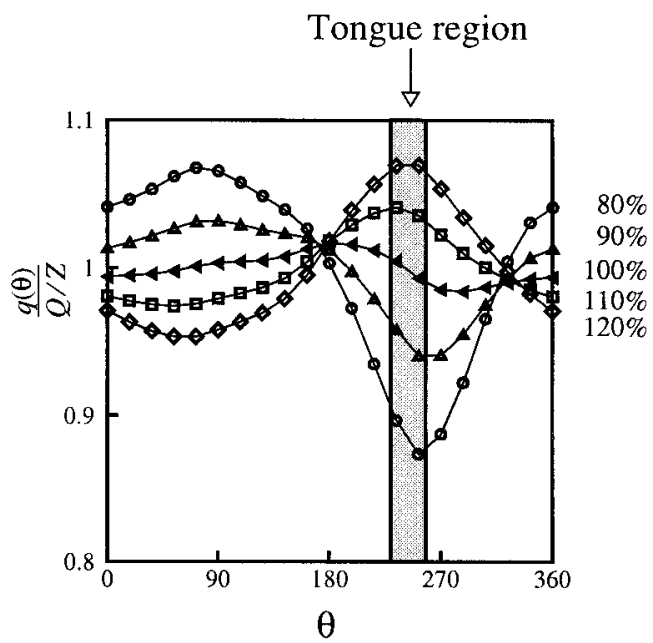

Figure 5. Comparison of the computations without and with unsteady wakes: variation of the flowrate $q(\theta)$ in a channel with orientation $\theta$ of the rotor blade (pressure side) for total flowrates of $80,90,100,110$ and $120 \%$ of the nominal flowrate. The flowrate in a channel is made dimensionless with (constant) total flowrate $Q$ and number of rotor blades $Z$. The region of the stator tongue is marked

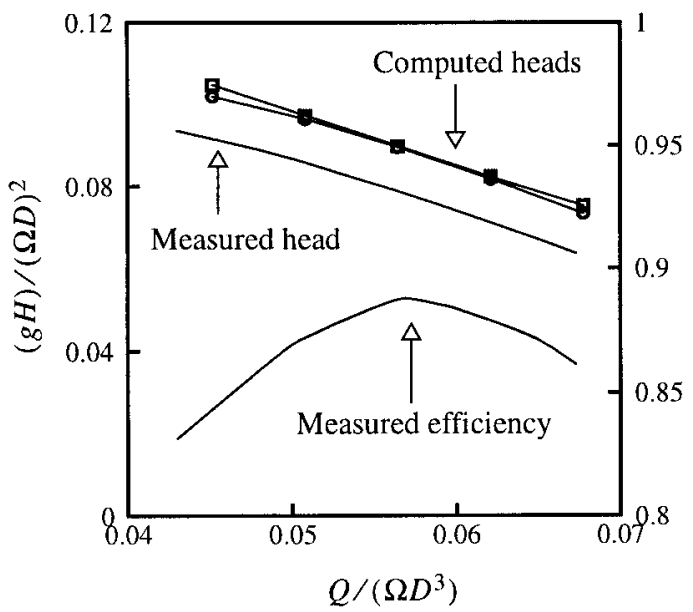

Figure 6. Computed and measured dimensionless head-capacity curves and measured overall efficiency $\eta$. Two computed head curves are shown: without unsteady wakes $(O)$ and with unsteady wakes $(\square)$. $D$ is the rotor diameter 
Currently the analysis system is being extended with models for the various (viscous) losses, such as volumetric, disc friction and hydraulic losses. ${ }^{20-22}$ This makes it feasible to predict the efficiency of complete pump configurations. It also results in more accurate $Q-H$ curves.

\section{CONCLUSIONS}

A very efficient numerical method was developed for the computation of unsteady, threedimensional potential flows in hydraulic pumps and turbines. The superelement method has been extended in order to eliminate slave DOFs not only from the governing Laplace equation, but also from the Kutta conditions. The resulting superelement formulation is invariant under rotation. Therefore the geometrical symmetry of the flow channels in the rotor can be exploited. This makes the method very suitable to performing fully coupled computations of the unsteady flow phenomena in both rotor and stator, the so-called rotor-stator interaction.

The developed numerical method was used to simulate the unsteady flow in an industrial mixed-flow pump. Two types of simulation are considered: one in which unsteady wakes behind the trailing edges of the rotor blades are taken into account and one in which these are neglected. Results are shown for the dependence of the blade circulation and the flowrate in a rotor channel on the orientation of the blade with respect to the stator tongue. These dependencies are measures of the importance of unsteady flow phenomena. Significant unsteady phenomena are observed. Simulations with unsteady wakes exhibit smaller variations than those without unsteady wakes. In both cases the variations increase with the deviation from the nominal flowrate. The overall head-capacity curve is hardly influenced by whether or not unsteady wakes are taken into account. The computations overestimate the head, since viscous losses are not accounted for in the basic potential flow model. The shape of the head-capacity curve is well predicted.

\section{APPENDIX: IMPLEMENTATION OF WAKE MODELS}

This appendix deals with some aspects of the implementation of the wake models. A detailed account is given in Reference 22. The value of the potential jump on the wake depends on the type of computation. Two types are distinguished: quasi-steady computations (without unsteady wakes) and unsteady computations (with unsteady wakes). The wake representation is shown in Figure 1. As explained below, these computations differ in the nature of the jump distribution on the wake: known vs. unknown jumps.

\section{Quasi-steady computations}

The flow field in a rotor-stator configuration of a pump will be unsteady. Especially at off-design conditions, the blade circulations will vary in time. One way of computing this unsteady flow would be to incorporate the variation of blade circulations along the span, but to neglect the convection of shed vortices in the wake. This is called the quasi-steady approach. If wake surfaces could extend from the trailing edges down to the exit pipe of the pump, the potential jumps would be described properly by (9). However, these surfaces cannot be extended beyond the cylindrical or conical rotor-stator interface. Therefore the varying potential jump on the wake must become 


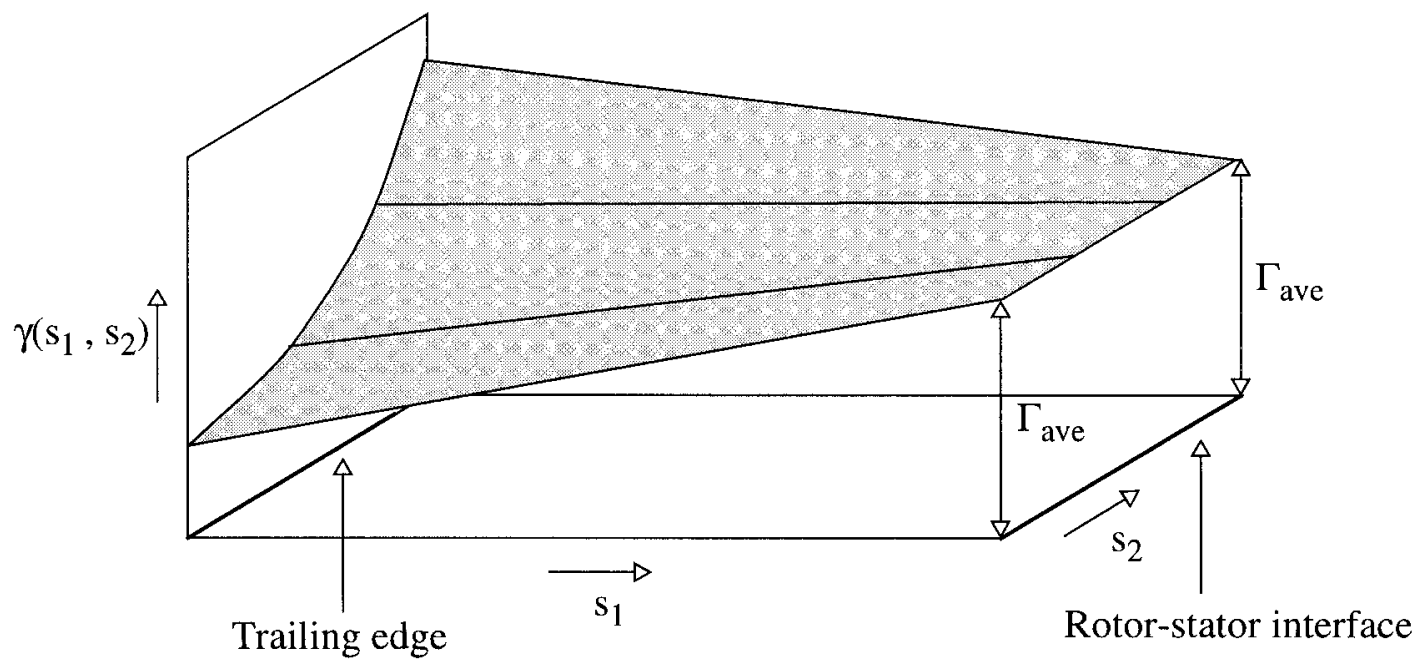

Figure 7. Schematic representation of the potential jump distribution $\gamma\left(s_{1}, s_{2}\right)$ on a wake in quasi-steady computations. The average blade circulation is $\Gamma_{\text {ave }}$

constant upon reaching this interface. In other words, the varying potential jump on the wake must eventually be averaged out. The value of the potential jump on the wake is now described by

$$
\gamma\left(s_{1}, s_{2}\right)=\Gamma\left(s_{2}\right)+\alpha\left(s_{1}, s_{2}\right)\left[\Gamma_{\text {ave }}-\Gamma\left(s_{2}\right)\right]
$$

where $\Gamma_{\text {ave }}$ is the spanwise average of the blade circulation. The factor $\alpha$ is dependent on the position in the wake and varies between the value 0 at the trailing edge and the value 1 at the rotor-stator interface. The second term on the right-hand side can be considered as a deviation from Kelvin's circulation theorem. However, when averaging this deviation over all wake nodes at constant $s_{1}$-co-ordinate, this averaged deviation reduces to zero. At the rotor-stator interface, the averaged value of the potential jump is guided to the outer wall of the pump, along part of the cylindrical or conical rotor-stator interface and some block boundaries located in the stator region of the pump. This type of averaging of the jump distribution is depicted in Figure 7.

In this type of wake modelling the jump distribution is determined completely by the unknown blade circulations.

\section{Unsteady computations}

Contrary to the former approach, a (fully) unsteady computation of the flow field in a rotorstator configuration requires the convection of vortices according to (8) to be taken into account as well. Once again, the varying potential jump will have to be averaged out at the rotor-stator interface. Suppose that the potential jump distribution at a given time step is given by $\gamma^{*}\left(s_{1}, s_{2}\right)$. An averaging procedure very similar to that for quasi-steady flow is now introduced:

$$
\gamma\left(s_{1}, s_{2}\right)=\gamma^{*}\left(s_{1}, s_{2}\right)+\alpha\left(s_{1}, s_{2}\right)\left[\gamma_{\text {ave }}^{*}\left(s_{1}\right)-\gamma^{*}\left(s_{1}, s_{2}\right)\right]
$$

where factor $\alpha$ depends on the position of the vortex in the wake and varies between the value 0 at the trailing edge and the value 1 at the rotor-stator interface. The average value of the potential 
jumps $\gamma^{*}\left(s_{1}, s_{2}\right)$ along the line with constant $s_{1}$ is denoted by $\gamma_{\text {ave }}^{*}\left(s_{1}\right)$. The deviation from the exact solution reduces to zero when averaging over nodes at constant $s_{1}$-co-ordinate. As was the case in the quasi-steady approach, the potential jump is equal to the local blade circulation at nodes on the trailing edge. Upon reaching the rotor-stator interface, the potential jump becomes equal for all $s_{2}$. This averaged value is guided to the outer wall of the pump.

In this type of wake modelling the jump distribution is determined by the unknown blade circulations and the known jump distribution on the wake corresponding to previously shed vortices.

\section{ACKNOWLEDGEMENT}

The authors would like to thank J. G. H. Op de woerd of BW/IP International Hengelo (PO Box 55, $7550 \mathrm{AB}$ Hengelo, The Netherlands) for providing the data on the geometry and performance characteristics of the BW/IP industrial mixed-flow pump.

\section{REFERENCES}

1. W. N. Dawes, 'A simulation of the unsteady interaction of a centrifugal impeller with its vaned diffuser: flow analysis', J. Turbomach., 117, 213-222 (1995).

2. D. Croba and J. L. Kueny, 'Numerical calculation of two-dimensional, unsteady flow in centrifugal pumps: impeller and volute interaction', Int. j. numer. methods fluids, 22, 467-482 (1996).

3. C. G. Speziale, 'Turbulence modelling in nonertial frames of reference', Theor. Comput. Fluid Dyn., 1, 3-19 (1989).

4. K. S. Chen and M. C. Sue, 'Finite element analysis of steady three-dimensional potential flow in the blade passage of a centrifugal turbomachine', Comput. Struct., 46, 625-632 (1993).

5. H. Daiguji, 'Numerical analysis of three-dimensional potential flow in centrifugal turbomachines', Bull. JSME, 26, 1495-1501 (1983).

6. B. Maiti, V. Seshadri and R. C. Malhotra, 'Analysis of flow through centrifugal pump impellers by finite element method', Appl. Sci. Res., 46, 105-126 (1989).

7. E. E. Morfiadakis, S. G. Voutsinas and D. E. Papantonis, 'Unsteady flow calculation in a radial flow centrifugal pump with spiral casing', Int. j. numer. methods fluids, 12, 895-908 (1991).

8. S. M. Miner, R. D. Flack and P. E. Allaire, 'Two-dimensional flow analysis of a laboratory pump', J. Turbomach., 114, 333-339 (1992).

9. R. Badie, J. B. Jonker and R. A. van den Braembussche, 'Finite element calculations and experimental verification of unsteady potential flow in a centrifugal pump', Int. j. numer. methods fluids, 19, 10831102 (1994).

10. J. B. Jonker and T. G. van Essen, 'A finite element perturbation method for computing fluid-induced forces on a centrifugal impeller rotating and whirling in a volute casing', Int. j. numer. methods eng., 29, 269-294 (1997).

11. G. K. Batchelor, An Introduction to Fluid Dynamics, Cambridge University Press, Cambridge, U.K., 1967.

12. O. C. Zienkiewicz and R. L. Taylor, The Finite Element Method, McGraw-Hill, Maidenhead, U.K., 1989.

13. E. Baskharone and A. Hamed, "A new approach in cascade flow analysis using the finite element method', AIAA J., 19, 65-71 (1981).

14. O. C. Zienkiewicz and J. Z. Zhu, 'The superconvergent patch recovery and a posteriori error estimates. Part I: the recovery technique', Int. j. numer. methods eng., 33, 1331-1364 (1992).

15. B. Hendrickson and R. Leland, 'An improved spectral graph partitioning for mapping parallel computations', SIAM J. Sci. Comput., 16, 452-469 (1995).

16. G. H. Paulino, I. V. M. Menezes, M. Gattass and S. Mukherjee, 'Node and element resequencing using the Laplacian of a finite element graph', Int. j. numer. methods eng., 37, 1511-1555 (1994). 
17. J. G. H. Op de woerd, Personal communication, 1995.

18. C. Pfleiderer, Die Kreiselpumpen für Flüssigkeiten und Gase, Springer-Verlag, Berlin, Germany, 1961.

19. N. P. Kruyt, B. P. M. van Esch and J. B. Jonker, 'Analysis of three-dimensional potential flows in centrifugal and mixed-flow pumps by a finite element method'. Proceedings 10th Conference on Fluid Machinery, 1995, Budapest, Hungary, pp. 242-251.

20. N. P. Kruyt, B. P. M. van Esch and J. B. Jonker, 'A tool for the analysis of unsteady potential flows in centrifugal and mixed-flow pumps'. Proceedings Pumpentagung 1996 C8-2, Karlsruhe, Germany, 1994.

21. B. P. M. van Esch, N. P. Kruyt and J. B. Jonker, 'An inviscid-viscous coupling method for computing flows in entire pump configurations'. Proceedings Third ASME Pumping Machinery Symposium, FEDSM97-3373, 1997, Vancouver, BC, Canada.

22. B. P. M. van Esch, 'Simulation of three-dimensional unsteady flow in hydraulic pumps'. Ph.D. Thesis, Department of Mechanical Engineering, University of Twente, The Netherlands, 1997. 